\title{
High-Dose Rate Brachytherapy
}

National Cancer Institute

\section{Source}

National Cancer Institute. High-Dose Rate Brachytherapy. NCI Thesaurus. Code C15651.

Internal radiation treatment that targets a cancerous tissue with accurate, high doses of radiation through the use of inserted temporary implants. 